\title{
Inkjet Printing of Controlled ZnO Nanoparticles Layering
}

\author{
N. Spinella*, C. Galati, L. Renna \\ STMicroelectronics, Stradale Primosole 50, 95121 Catania, Italy \\ E-mail: natalia.spinella@st.com
}

Received: 13 March 2019; Accepted: 31 March 2019; Available online: 10 May 2019

\begin{abstract}
Controlled layering of functional material can produced a versatile film with specific chemical and physical proprieties for desirable applications. This article presented inkjet multilayer structures of $\mathrm{ZnO}$ nanoparticles of specific layer morphology and thickness for the development of devices where a high surface-tovolume ratio is required (e.g. micro gas sensors). Stacked multilayers were stratified through a multi-run printing process suitable to produce large-square pattern on flat silicon support. The formation of a multilayer structure was demonstrate through an extended structural characterization of the resulting film. Printed layer morphology was investigated with optical and scanning electron microscopies; atomic force microscopy profiling characterizations were conducted over the entire printed area to evaluate the pattern reproducibility. Finally, a preliminary study as gas sensing film was performed, using the alcohol/ZnO interaction experiments.
\end{abstract}

Keywords: Inkjet printing; $\mathrm{ZnO}$ nanoparticles; Stacked-multilayers.

\section{Introduction}

Multilayer structures are desirable for developing advanced devices because they offer the opportunity to create a versatile film with specific chemical and physical proprieties. A wide range of functional materials were successfully prepared through solution processing techniques [1-3], however, the dipping steps, including in this approach, critically hamper their implementation in a real manufacturing process and consequentially the potential technological applications of the functional material. The inkjet method has recently demonstrated exceptional performances to stratify materials creating advanced nanocomposite with specific geometry and functionalities $[4,5]$. Moreover, respect to traditional methods it offers the opportunity for fast, practical and low-cost approaches even over an extended range of area and on a wide variety of substrates, including plastic materials [6-11]. The ability to print patterns with a higher resolution than other solution processing techniques has been widely documented too [12] together with the capability of defining structures without using any masks. These features demonstrate that the technique is useful for developing fully integrated and packaged electronic devices $[13,14]$ as well as for manufacturing flexible and wearable components for emerging technologies [15]. The inkjet printing process for thin film preparation allows the deposit of nanoparticle-based ink for the preparation of densely packed layers with appropriate thickness and composition for various applications [16] and well defined printed morphologies of nanoparticles can be already found in several works [17-19].

An inkjet multilayer structure of $\mathrm{ZnO}$ nanoparticles which preserves a specific layer morphology and a predictable thickness over extended support area is presented herein. $\mathrm{ZnO}$ is a leading semiconductor oxide that has gained wide usage in the semiconductor industry [20-22]. Recently, the ability of $\mathrm{ZnO}$ to easily form nanostructures offers researchers ideal morphologies for the development of devices where a high surface-tovolume ratio is required (gas sensing layer, catalyst, etc.) [23-25]. In this context, appropriate formulations of $\mathrm{ZnO}$ nanoparticulate inks were used to prepare stratified layers through a multi-run printing process suitable to produce large-square pattern on a flat silicon support. The formation of a stacked multilayer structure was obtained by a fine tuning of the printing parameters and demonstrate through an extended structural characterization of the resulting film. In particular, printed layer morphology was investigated with optical microscopy, Atomic Force Microscopy and Scanning Electron Microscopy. AFM profiling was performed over the entire printed area to determine the pattern reproducibility. Focused ion beam cross-sections relative to specific structural features were prepared and analyzed to evaluate the layer thickness. The sensing properties of the inkjet multilayer were finally evaluated for the ethanol detection using a silicon micromachined micro hotplate device [25].

\section{Experimental}

\subsection{Materials}


The $\mathrm{ZnO}$ nanoparticles inks were prepared by Avantama AG. The ink viscosity and surface tension were formulated to match the fluid requirements for the inkjet printing process. The nominal size of the $\mathrm{ZnO}$ nanoparticles was $\sim 15 \mathrm{~nm}$, as indicated by the supplier.

\subsection{Preparation of $\mathrm{ZnO}$ layer}

ZnO layers were prepared using a FUJIFILM Dimatix Materials printer (DMP-2800 series) operating with a single nozzle. The supplied $\mathrm{ZnO}$ ink was filtered with $0.2 \mu \mathrm{m}$ filter paper, sonicated for several minutes and dispensed with a $10 \mathrm{pL}$ nominal drop-volume Dimatix cartridge. Filled cartridges were stored at room temperature until use. Square-shaped patterns of $1 \mathrm{mmx} 1 \mathrm{~mm}$ were printed on silicon supports pre-heated to $60^{\circ} \mathrm{C}$ by single or multi-pass printing procedures. The last was performed with a delay time of $20 \mathrm{~s}$ for each layer. Once printed, the samples were analyzed without any further treatments or processes.

\subsection{Characterization methods}

Optical microscopy (MX50) was used for the initial inspection of the layer structure. Surface morphology was further analyzed by AFM (Veeco VX210) and SEM (ZEISS NEON 40). For thickness evaluation, sample crosssections of specific pattern regions were prepared by an in situ Focused Ion Beam procedure performed with the same SEM equipment. AFM was also used for measuring the surface profiles of the printed pattern.

\section{Results and discussion}

\subsection{Role of printing parameters}

A detailed set-up of the operative conditions is required for a multilayer design, and even though fine tuning must be performed on the specific structured device, an understanding of the effect of the parameters role could be figured out on a flat substrate with similar surface properties. In order to form nanoparticle $\mathrm{ZnO}$ layers with specific geometries and controlled thickness, appropriate test patterns over a flat silicon support were designed in order to study and optimize the principal parameters affecting printing quality. Among these, the drop spacing (DS), defined as the center-to-center drop distance, was adjusted to achieve the optimal distance at which the coalescence of jetted drops forms a well-defined square pattern of approximately $1 \mathrm{~mm}$ length.

The firing voltage was initially adjusted to jet drops with a speed of approximately $10 \mathrm{~m} / \mathrm{s}$ at a jetting frequency of $5 \mathrm{kHz}$. Under these conditions the jetted drop vertically exits from the nozzle, and a flying round drop was formed at a distance that matches the print-head to substrate distance $(1000 \mu \mathrm{m})$, figure 1 .

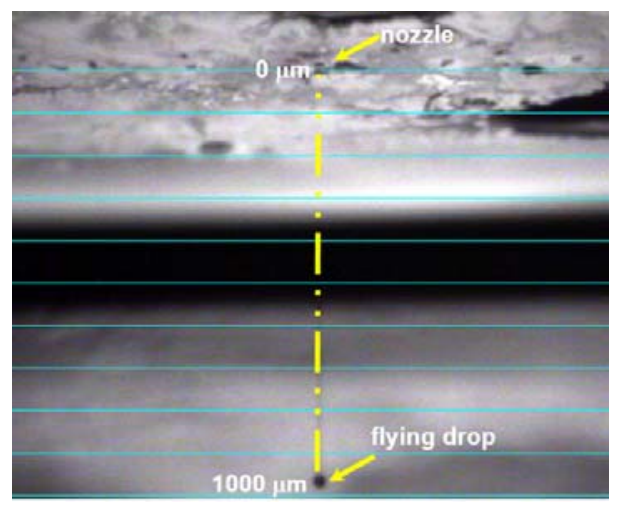

Figure 1. Dimatix watcher image of the jetted drop from a nozzle.

Platen temperature is also a significant factor. In addition to the stated experimental conditions, the printed platen was heated to $60^{\circ} \mathrm{C}$ to avoid spreading and undesired coalescence effects and ensure fast drying of the drop during the entire process.

A DS value large enough to guarantee physical separation (e.g. $100 \mu \mathrm{m}$ ) through a single-pass process of arrayed $\mathrm{ZnO}$ drops was first used. Figure 2(a) demonstrates that this condition leads to the formation of isolated round drops around 80-90 $\mu \mathrm{m}$ in diameter, spaced at approximately $100 \mu \mathrm{m}$. As shown in the picture, the resulting array is aligned and the coffee ring effect observed elsewhere [16, 26, 27] was not present; a tendency of the fluid to pool at the center of the drop was instead noticed. Even if some random coalescence is potentially observable even in the single-run drop array, an evident coalescence phenomenon appears by printing the same pattern four-time with a sufficient delay for the drops to dry between each run, figure 2(b). The resulting array presents several rows where two or more subsequent drops coalesce into a single-horizontal strip along the printing direction indicated 
by the arrow. As also previously described in Soltman [27], the landing drops in this experiment overlap and merge preserving their round shape, and a scalloped printed line emerges.

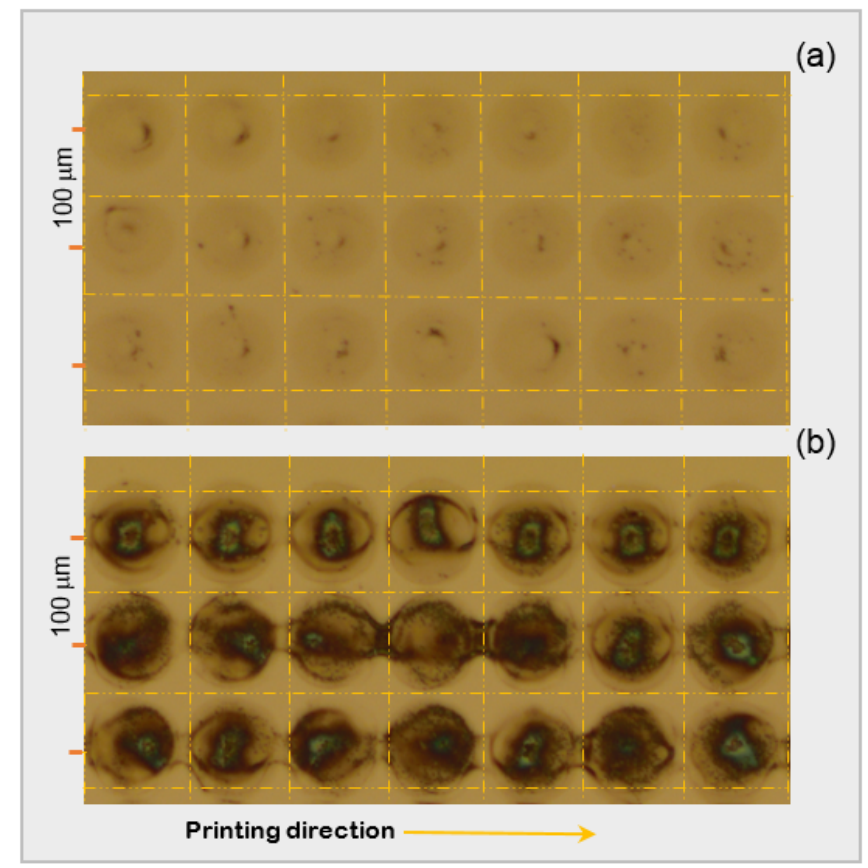

Figure 2. Optical images of $\mathrm{ZnO}$ drop array printed by a single (a) and a four-pass process (b), DS $100 \mu \mathrm{m}$. Grid step is $85 \mu \mathrm{m}$.

Considering a drop size of approximately $85 \mu \mathrm{m}$, a continuous square coverage $1 \mathrm{~mm}$ in size should be formed at a DS value equal to $60 \mu \mathrm{m}$. At this DS value, the relative printed patterns have pseudo-square structures, both following a single-pass and a four-pass printing process, figure 3(a) and (b) respectively, consisting of a sequence of horizontally-oriented strips.

A smaller drop spacing reduces printing quality with severe coalescence causing amorphous structures from an overflowing irregular drop (data not shown).

(a)

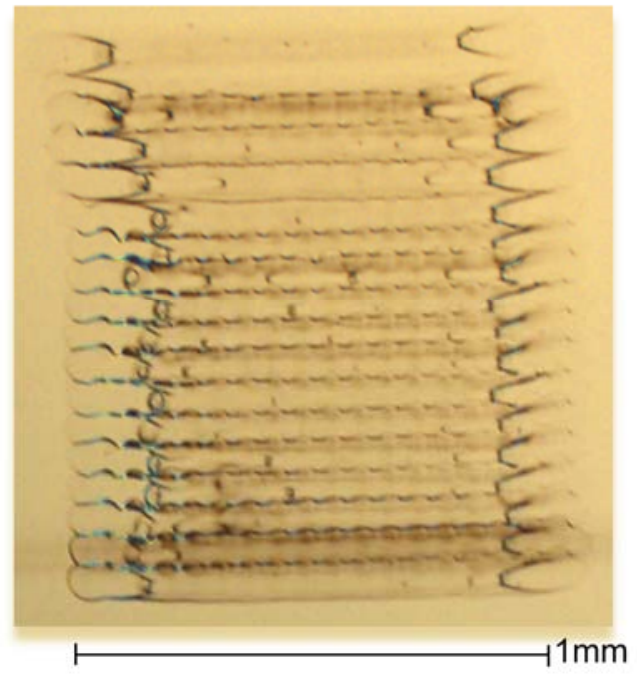

(b)

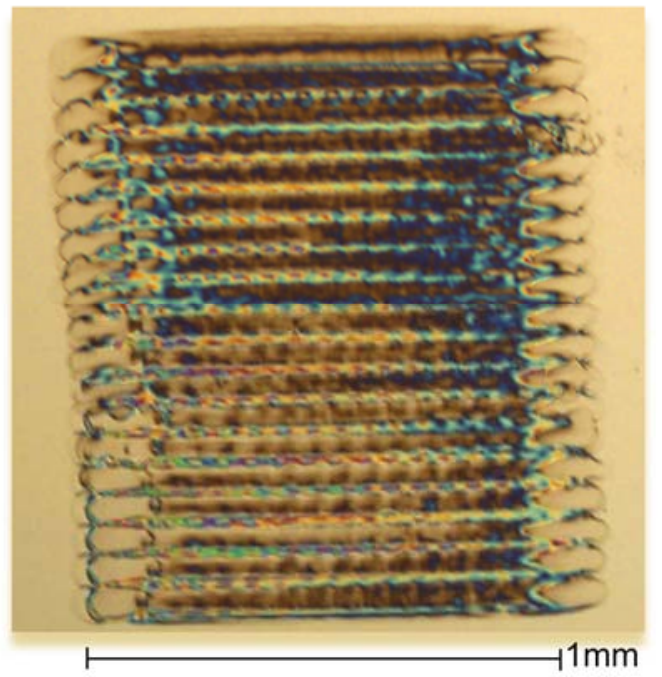

Figure 3. Optical images of 1mmx1mm inkjet single (a) and four-pass $\mathrm{ZnO}$ layers (b), DS $60 \mu \mathrm{m}$.

\subsection{Structural analysis}

Initial inspection of the single-layer pattern printed at a DS $60 \mu \mathrm{m}$ reveals wave shaped strips which are aligned well with each other and show minimum (or maximum) points at a distance that is very close to the imposed DS 
value, figure 4(a). In addition, the wave shape is coherent with the drop size, as described in the sketch of the printing layout. In accordance with the coalescence effect shown in figure 2(b), the landing drops overlap and coalesce preferentially in the printing direction, preserving their round shape; this time, however, the scalloped morphology shows that all the concavities are oriented at the same direction as a consequence of the overlap among two subsequent printing lines.

The strip structure is preserved even after the four-pass printing process; in this case, the strips do not form a clear wave pattern, but straighter and larger strip boundaries. The inner strip region is narrower (approximately 40 $\mu \mathrm{m}$ in size) with respect to the (yellow double row) distance of the single dispensation (figure 4a), while the boundary-width, taken as the vertical distance between two subsequent inner-strips, is approximately $18 \mu \mathrm{m}$. Therefore, the overall strip size is still coherent with the size of a single drop, figure 4(b), which consistent with the formation of a stack of aligned straight strips that emerges from the controlled drop overlap that occurs in each printing step.

(a)

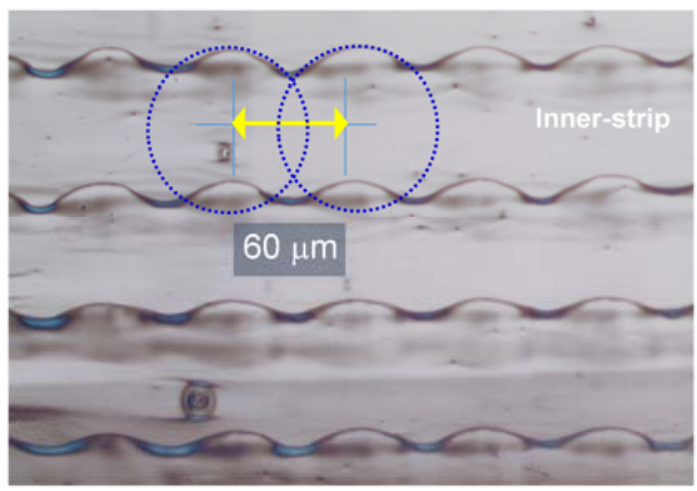

(b)

Figure 4. Optical images relative to the central area of $\mathrm{ZnO}$ single (a) and four-layers patterns (b); a sketch of the printing layout at $60 \mu \mathrm{m}$ drop spacing for an $85 \mu \mathrm{m}$ sized drop is also shown in (a).

The AFM image of the single-layer printed pattern, figure 5(a), shows a relatively smooth surface for the inner strip region characterized by a low root-mean square roughness value $(\mathrm{Rq}=3.5 \mathrm{~nm})$ and less oscillation in height, figure 5(b); the top of the wave instead reaches a maximum height of approximately $150 \mathrm{~nm}$. The nanoparticulate nature of the printed layers was examined through SEM analysis. As shown in figure 5(c) the printed pattern forms a continuous and densely packed coverage without severe cracks $(<100 \mathrm{~nm})$, consisting of a homogenous distribution of $\mathrm{ZnO}$ nanoparticles (approximately $10-15 \mathrm{~nm}$ in size).

(a)
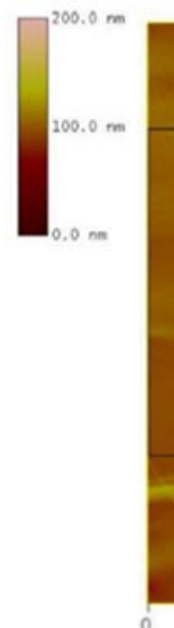

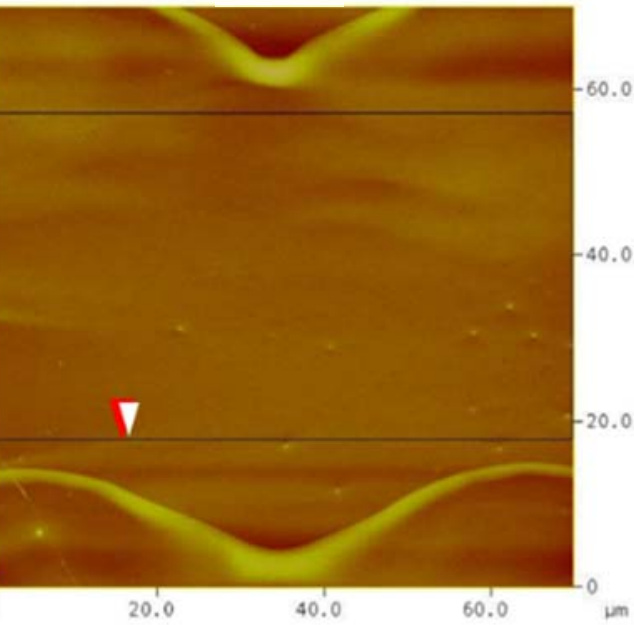

(b)

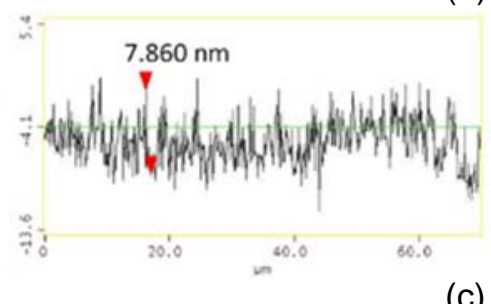

(c)

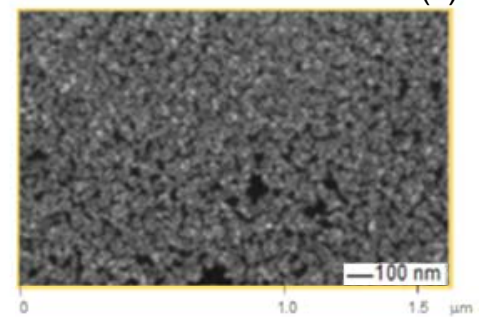

Figure 5. AFM image of the $\mathrm{ZnO}$ single layer pattern (a) and relative section analysis measured along the marked black line (b). SEM image of the inner strip region (c).

\subsection{Controlling the layer thickness}


In order to evaluate the layer thickness, appropriate cross-sections of the printed patterns were obtained through an in situ FIB preparation and analyzed with high-resolution SEM imaging. Figure 6 shows the FIB sections relative to internal strip zones. Ion irradiation may alter the observed section morphology, which can result in poor image contrast especially for the thinner layers. For the four-layer sample, it was in any case possible to evaluate the layer thickness and several section zones with relatively smooth profiles have shown values of about $140 \mathrm{~nm}$. Increasing the number of layers results in thicker film that still preserve a relative uniform thickness in the inner strip region; the thickest film, prepared through a twelfth run process, have presented a predictable thickness of approximately $441 \mathrm{~nm}$ (data not shown).

(a)
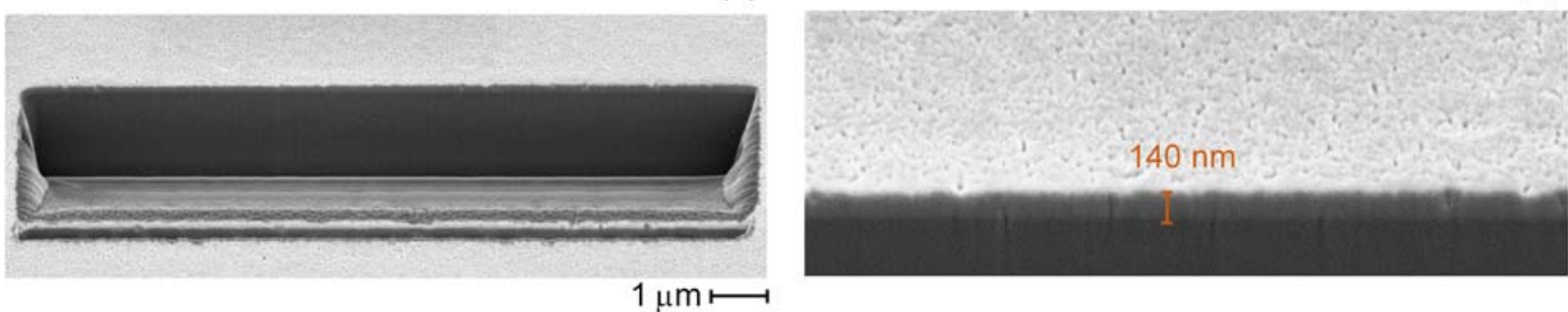

Figure 6. SEM analysis relative to the FIB cross-section of the four-layers $\mathrm{ZnO}$ film (a); in (b) a SEM magnification relative to the same section.

In figure 7 is presented the AFM profiling measured long an entire deposited line of the single and four-layer film, (a) and (b) respectively, it demonstrates the good reproducibility of the strips, which replicated each other well along the printed area (see double arrows in figure 7), closely matching the imposed drop spacing value (60 $\mu \mathrm{m})$. In addition, the broadening of the strip boundary clearly appears for each strip of the four-layer pattern, showing a larger peak width of approximately $20 \mu \mathrm{m}$ in figure $7(\mathrm{~b})$ with respect to the sharp peaks for the single layer in figure 7(a). Regarding the roughness with respect to the section analysis previously discussed on a small area (see figure 5), on a very large area, the internal strip region preserves low height oscillations $(\sim 10 \mathrm{~nm})$ and relative smooth morphologies, especially for the single-layer pattern.
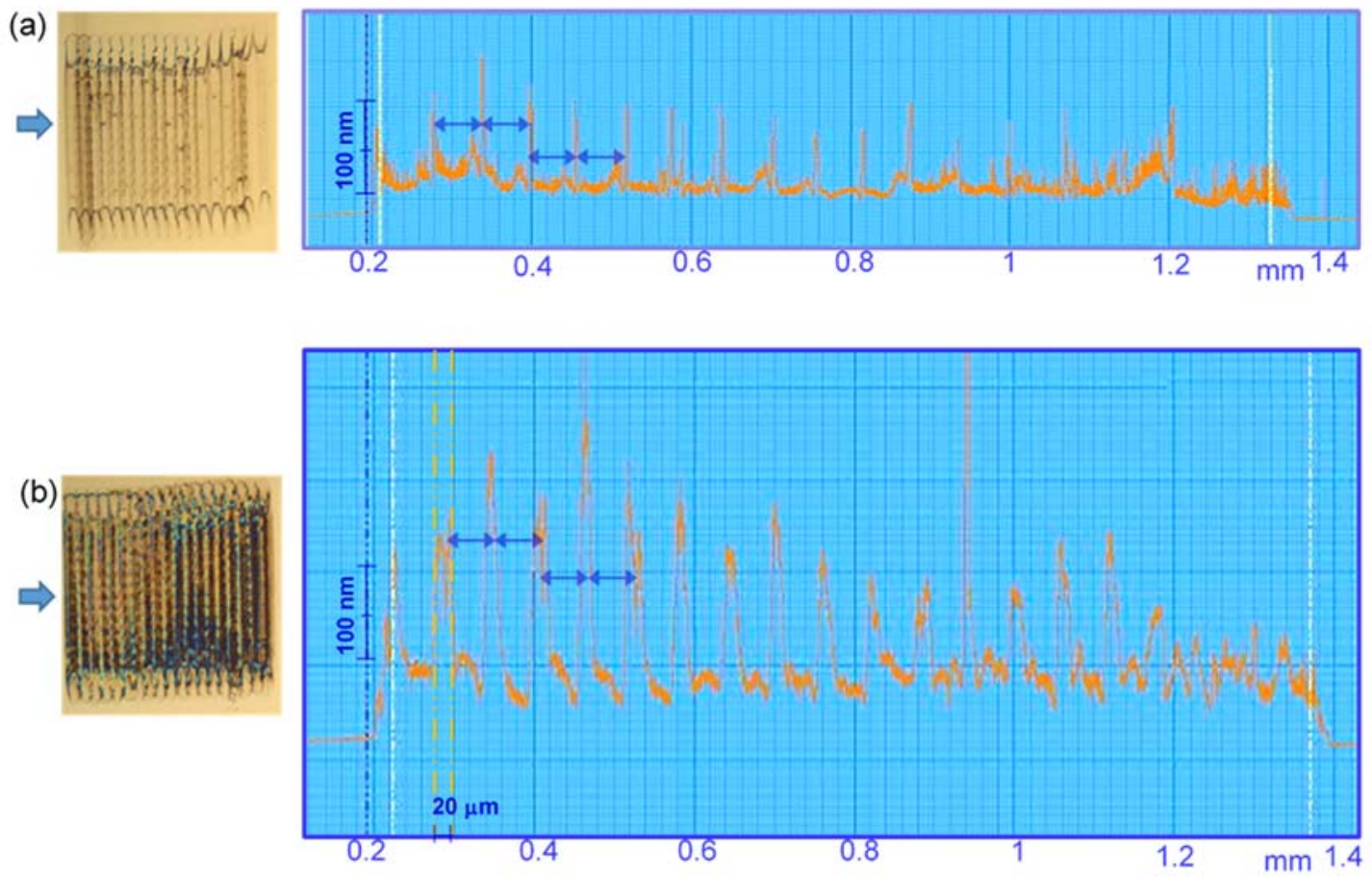

Figure 7. AFM height profiling of the single-layer (a) and four-layer printed patterns (b), and the relative optical images and scanning directions. The peak-to-peak distance is indicated by the double-arrows, with a length equal to $60 \mu \mathrm{m}$. 
The sensing properties of the $\mathrm{ZnO}$-based inkjet film were evaluated creating stacked structures on a siliconbased 400x400 $\mathrm{m}^{2}$, highly integrated micro hotplate for ethanol sensing, early described in [25]. Preliminary experiments have presented a sensing response of about $50 \%$ as well as a reproducibly and consistent variation to the ethanol flux. This results demonstrate the potential application of the stacked inkjet structure as gas sensing layer in micro devices; enhancing of the sensor performances can be achieved by a fine adjusting of the experimental conditions (e.g. operating temperature, environmental humidity, platen temperature etc.) and depositing a convenient material amount respect to the number of printed layers. In addition, further post printing treatments can be implemented to enhance the sensing material proprieties.

\section{Conclusion}

Stacked multilayers of $\mathrm{ZnO}$ nanoparticles with smooth strips of specific thickness are produced through a fast, practical and low-cost inkjet printing process. The role of the printing parameter was thoroughly examined and fine adjustments were applied accordingly. A continuous sequence of strips with periodical repeats over a large square area was accomplished. Very small $\mathrm{ZnO}$ nanoparticles-based inks formulated with appropriate viscosity and surface tension were chosen for their wide ability to form nanostructures with high surface-to-volume ratio for the applications that require this feature.

As a proof of concept, the gas sensing properties of the inkjet multilayer were investigated by using a highly integrated silicon micro-heater. Further work is underway to enhance the sensing performances by a rational choice of the operative conditions, including various particle solutions to accomplish innovative nanostructured sensing materials.

\section{References}

[1] Lisdat F. Trends in the layer-by-layer assembly of redox proteins and enzymes in bioelectrochemistry. Current Opinion in Electrochemistry. 2017;5(1):165-172.

[2] Sato K, Anzai JI. Dendrimers in layer-by-layer assemblies: synthesis and applications. Molecules. $2013 ; 18(7): 8440-8460$.

[3] Liu Z, Bai L, Zhao G, Liu Y. Sandwich-like layer-by-layer assembly of gold nanoparticles with tunable SERS properties. Beilstein journal of nanotechnology. 2016;7(1):1028-1032.

[4] Andres CM, Kotov NA. Inkjet deposition of layer-by-layer assembled films. Journal of the American Chemical Society. 2010;132(41):14496-14502.

[5] Choi M, Heo J, Choi D, Hwangbo S, Hong J. Inkjet Printing Based Layer-by-Layer Assembly Capable of Composite Patterning of Multilayered Nanofilms. Macromolecular Materials and Engineering. $2017 ; 302(12): 1700332$.

[6] Forrest SR. The path to ubiquitous and low-cost organic electronic appliances on plastic. Nature. 2004, 428 (6986): 911-918.

[7] Van Osch TH, Perelaer J, de Laat AW, Schubert US. Inkjet printing of narrow conductive tracks on untreated polymeric substrates. Advanced Materials. 2008;20(2):343-345.

[8] Uttiya S, Bernini C, Vignolo M, Pallecchi I, Marré D, Siri AS, Pellegrino L. Inkjet printing of conducting silver patterns on alumina and insulating ceramic-glass by saline precursors. Thin Solid Films. 2017;642:370376.

[9] Basiricò L, Cosseddu P, Fraboni B, Bonfiglio A. Inkjet printing of transparent, flexible, organic transistors. Thin Solid Films. 2011;520(4):1291-1294.

[10] Lilliu S, Böberl M, Sramek M, Tedde SF, Macdonald JE, Hayden O. Inkjet-printed organic photodiodes. Thin Solid Films. 2011;520(1):610-615.

[11] Scandurra A, Francesco Indelli G, Graziana Sparta N, Galliano F, Ravesi S, Pignataro S. Low-temperature sintered conductive silver patterns obtained by inkjet printing for plastic electronics. Surface and Interface Analysis. 2010;42(6-7):1163-1167.

[12] Gamerith S, Klug A, Scheiber H, Scherf U, Moderegger E, List EJ. Direct ink-jet printing of Ag-Cu nanoparticle and Ag-precursor based electrodes for OFET applications. Advanced Functional Materials. 2007;17(16):3111-3118.

[13] Farooqui MF, Karimi MA, Salama KN, Shamim A. 3D-Printed Disposable Wireless Sensors with Integrated Microelectronics for Large Area Environmental Monitoring. Advanced Materials Technologies. 2017;2(8):1700051.

[14] Guo F, Li N, Radmilović VV, Radmilović VR, Turbiez M, Spiecker E, Forberich K, Brabec CJ. Fully printed organic tandem solar cells using solution-processed silver nanowires and opaque silver as charge collecting electrodes. Energy \& Environmental Science. 2015;8(6):1690-1697. 
[15] Singh R, Singh E, Nalwa HS. Inkjet printed nanomaterial based flexible radio frequency identification (RFID) tag sensors for the internet of nano things. RSC Advances. 2017;7(77):48597-48630.

[16] Shen W, Zhao Y, Zhang C. The preparation of ZnO based gas-sensing thin films by ink-jet printing method. Thin Solid Films. 2005;483(1-2):382-387.

[17] Vaseem M, Lee KM, Shin JK, Hahn YB. Synthesis of ZnO nanoparticles and their ink-jetting behavior. Journal of nanoscience and nanotechnology. 2012;12(3):2380-2386.

[18] Kang JS, Kim HS, Ryu J, Hahn HT, Jang S, Joung JW. Inkjet printed electronics using copper nanoparticle ink. Journal of Materials Science: Materials in Electronics. 2010;21(11):1213-1220.

[19] Chen CT, Hung TY. Morphology and deposit of picoliter droplet tracks generated by inkjet printing. Journal of Micromechanics and Microengineering. 2016;26(11):115005.

[20] Klingshirn C. ZnO: From basics towards applications. physica status solidi (b). 2007;244(9):3027-3073.

[21] Özgür Ü, Alivov YI, Liu C, Teke A, Reshchikov M, Doğan S, Avrutin VC, Cho SJ, Morkoç H. A comprehensive review of ZnO materials and devices. Journal of applied physics. 2005;98(4): 041301.

[22] Arnold MS, Avouris P, Pan ZW, Wang ZL. Field-effect transistors based on single semiconducting oxide nanobelts. The Journal of Physical Chemistry B. 2003;107(3):659-663.

[23] Feng P, Wan Q, Wang TH. Contact-controlled sensing properties of flowerlike ZnO nanostructures. Applied Physics Letters. 2005;87(21):213111.

[24] Chang CJ, Hung ST, Lin CK, Chen CY, Kuo EH. Selective growth of ZnO nanorods for gas sensors using ink-jet printing and hydrothermal processes. Thin Solid Films. 2010;519(5):1693-1698.

[25] Alberti A, Renna L, Sanzaro S, Smecca E, Mannino G, Bongiorno C, Galati C, Gervasi L, Santangelo A, La Magna A. Innovative spongy $\mathrm{TiO} 2$ layers for gas detection at low working temperature. Sensors and Actuators B: Chemical. 2018;259:658-667.

[26] Lee DJ, Oh JH. Shapes and morphologies of inkjet-printed nanosilver dots on glass substrates. Surface and interface analysis. 2010;42(6-7):1261-1265.

[27] Soltman D, Subramanian V. Inkjet-printed line morphologies and temperature control of the coffee ring effect. Langmuir. 2008;24(5):2224-2231.

(C) 2019 by the author(s). This work is licensed under a Creative Commons Attribution 4.0 International License (http://creativecommons.org/licenses/by/4.0/). Authors retain copyright of their work, with first publication rights granted to Tech Reviews Ltd. 